\title{
ENFOQUE SITUACIONAL Y TUTORÍA ACADÉMICA COMO ESTRATEGIA FORMATIVA EN ASIGNATURAS PRÁCTICAS DE TRABAJO SOCIAL
}

\section{SITUATIONAL APPROACH AND ACADEMIC TUTORING AS A TRAINING STRATEGY IN PRACTICAL SUBJECTS IN THE SOCIAL WORK PROGRAM}

Fecha recepción: agosto de 2019 / fecha aceptación: noviembre de 2019

\section{Marcela Concha Toroํㅗ Marcela Muñoz Cuevas² y Yasna Anabalón Anabalón ${ }^{3}$}

Cómo citar este artículo:

Concha, M., Muñoz, M., y Anabalón, Y. (2019). Enfoque situacional y tutoría académica como estrategia formativa en asignaturas prácticas de trabajo social. Revista Pensamiento y Acción Interdisciplinaria, 5(2), 64-83. DOI: http://doi.org/10.29035/pai.5.2.64

\begin{abstract}
Resumen
Las universidades chilenas adscritas al Consejo de Rectores, han abordado diversas iniciativas en función de la innovación curricular, asumiendo desafíos internacionales de formación en los contextos de desempeño profesional. En el escenario de las prácticas profesionales, las tutorías académicas, adquieren relevancia en la formación disciplinaria, considerando el enfoque situacional como una forma de interpretar y aproximarse a la realidad reconociendo las claves discursivas del problema social a partir de una situación. Este artículo presenta el enfoque situacional y la tutoría académica como estrategia formativa en las asignaturas de práctica profesional de la Escuela de Trabajo Social perteneciente a la Universidad del Bío-Bío, Chile. La experiencia muestra que trabajar desde este enfoque en las prácticas profesionales implica establecer, de acuerdo al nivel curricular en el que se encuentre el estudiante, una serie de actividades y procedimientos que se irán implementando por parte de los tutores de práctica durante el tiempo que se desarrolla el proceso, facilitando la experiencia y el aprendizaje del enfoque de intervención a través de la tutoría académica como estrategia formativa.
\end{abstract}

Palabras claves: formación inicial, trabajo social, tutoría, sistema de práctica, universidad.

\footnotetext{
1 Trabajadora Social, chilena. Magister en Salud Pública con mención en Salud Familiar, Académica Escuela de Trabajo Social Universidad del Bío-Bío, Chillán, Chile. Correo electrónico: maconcha@ubiobio.cl.

2 Asistente Social, chilena. Licenciada en Trabajo Social, Magister en Desarrollo Humano Local y Regional, Coordinadora de Prácticas de la Carrera de Trabajo Social de la Universidad del Bío-Bío, Concepción, Chile. Correo electrónico: memunoz@ ubiobio.cl.

3 Trabajadora Social, chilena. Magister en familia con mención en mediación familiar y Candidata a Doctora en educación en Consorcio, perteneciente de la Universidad del Bío-Bío. Becaria CONICYT/ Doctorado Nacional/ 2017/ Folio 21170133. Tutora de práctica de la Escuela de Trabajo Social, Universidad del Bío-Bío, Chillán, Chile. Correo electrónico: yanabalon@ ubiobio.cl.
} 


\begin{abstract}
Chilean universities attached to the Council of Rectors have addressed various initiatives in terms of curriculum innovation, assuming international training challenges in the contexts of professional performance. In the scenario of professional practices, academic tutoring acquires relevance in disciplinary training, considering the situational approach as a way of interpreting and approaching reality by recognizing the discursive keys of the social problem from a situation. This article presents the situational approach and academic tutoring as formative strategy in the professional practice subjects of the Social Work Program belonging to the Universidad del Bío-Bío, Chile. Experience shows that working from this approach in professional practices involves determining, according to the curricular level in which the student is, a series of activities and procedures that will be implemented by the practice tutors during the time in which the process is developed, providing experience and learning of the intervention approach through academic tutoring as a training strategy.
\end{abstract}

Keywords: Initial training, social work, tutoring, practice system, university.

\title{
Introducción
}

Considerando los lineamientos y coyuntura de la educación universitaria a nivel internacional, post Bolonia, y también a nivel nacional, se reconceptualiza el rol que asume la formación por competencias como eje del diseño curricular de las carreras de pre-grado, lo cual implica tal como lo presentan Inostroza, Zúñiga y Ayala (2013), modificar en dos niveles el sistema formativo: a.- rediseñar la estructura curricular, permitiendo evidenciar el cambio de formación por objetivos a uno basado en competencias, y la interrelación de los contenidos en función de un conjunto de habilidades (Tardif, 2003), y b.- cambiar el enfoque pedagógico centrado en la enseñanza del docente, hacia uno centrado en el estudiante (Tejada, 2002).

Por otra parte, es necesario generar cambios en el currículum, puesto que surgen nuevos enfoques en las disciplinas, producto de hallazgos de investigaciones, como también actualizaciones del punto de vista tecnológico y junto a ello, nuevas prácticas basadas en metodologías activas, que implica generar un tránsito del currículum academicista a uno centrado en el aprendizaje, tal como lo establecen los modelos educativos universitarios, concebidos para implementar estos nuevos cambios de cómo reconceptualizar el aprendizaje y la enseñanza. Por tanto, la necesidad de transformar los itinerarios formativos, tal como lo establecen (Organización para la Cooperación y el Desarrollo Económicos [OCDE], 1998; Delors, 1996; Rollin, 1997; Pey, Chauriye y Chiuminatto, 2018; Bernasconi y Rojas, 2004; Consejo de Rectores de Universidades chilenas [CRUCH], 2013), es propio de sistemas que reflexionan sobre su misión y visión formativa, caracterizados por poseer capacidad de transformarse (CRUCH, 2012). 
Bajo este contexto, las universidades chilenas adscritas al Consejo de Rectores, han abordado diversas iniciativas en función de la innovación curricular (CRUCH, 2012), asumiendo desafíos internacionales de la formación profesional, cuya naturaleza es compleja y dinámica.

Es así como, en el escenario de las prácticas profesionales, la tutoría académica adquiere relevancia en la formación disciplinaria, que para la Escuela de Trabajo Social de la Universidad del Bío-Bío, consiste en un proceso de acompañamiento profesional y personal al estudiante durante todo el periodo de prácticas, considerando los ámbitos académico, personal, social y profesional, siendo realizada por docentes dedicados a esta labor, que son denominados tutores de práctica (Anabalón, Concha y Mora, 2018).

El presente artículo tiene por objetivo comunicar y analizar la estrategia formativa de la tutoría académica bajo el enfoque situacional, que se desarrolla en las asignaturas de práctica profesional de la Escuela de Trabajo Social de la Universidad del Bío-Bío.

En cuanto a su contenido, se resaltan los elementos teóricos que sustentan el modelo de formación adoptado por esta Escuela y cómo se expresa en las asignaturas de práctica profesional; los diversos instrumentos para el análisis situacional, el diseño, la evaluación, el registro y la reflexión que se utilizan en este contexto, así como también los niveles y momentos de la práctica profesional, para finalizar con el rol y las fases de la tutoría académica.

\section{Desarrollo}

La carrera de Trabajo Social de la Universidad del Bío-Bío, no ha estado ajena a estos cambios, pues como parte de la Red de Escuelas de Trabajo Social del $\mathrm{CRUCH}$, en el año 2003 participó de un proyecto sobre Mejoramiento de la Calidad de la Educación Superior (MECESUP) denominado Innovación curricular desde el enfoque de competencias: El nuevo desafío de las Escuelas de Trabajo Social del Consejo de Rectores, cuyo propósito fue mejorar la formación profesional de los estudiantes de las escuelas de Trabajo Social pertenecientes a las Universidades del Consejo de Rectores constituyentes del consorcio, a partir de un currículum basado en competencias, fortalecimiento de las capacidades de docencia e innovación tecnológica y movilidad estudiantil a fin de generar condiciones para una futura empleabilidad exitosa (Universidad del Bío-Bío, 2012). En consecuencia y producto de lo anterior, surge la importancia de generar nuevas propuestas curriculares en la formación de competencias genéricas y específicas o disciplinarias, que se amalgaman para formar el perfil de egreso de los Trabajadores/as Sociales.

Por tanto, desde el punto de vista curricular, el equipo académico de la Escuela, toma la decisión de efectuar cambios a la luz de un proceso de autoevaluación y 
diagnóstico previo que arroja falencias en la formación, nuevas tendencias, entre otros aspectos, conjunción de elementos que da paso a la formulación de un perfil de egreso en el que se distingue al profesional por desarrollar procesos de intervención social, fundados, situados y reflexivos en el marco de las ciencias sociales y humanas, para lo cual cuenta con una sólida formación en investigación social que permite comprender y explicar las distintas realidades sociales, favoreciendo la construcción de dispositivos de intervención para distintos niveles de complejidad, resguardando el desarrollo humano integral y la promoción de los derechos humanos, con especial énfasis en el nivel regional y nacional (Universidad del Bío-Bío, 2012). Es así que este perfil se encuentra compuesto por tres grandes competencias que dicen relación con un conjunto de habilidades que el estudiante de la carrera de Trabajo Social debe evidenciar que aprendió en el área de fundamentos teóricos, investigación e intervención social.

Consecuentemente bajo este contexto, y desde la perspectiva curricular, las prácticas profesionales se sitúan en la línea o área formativa de intervención social, promoviendo el desarrollo de competencias específicas y sub-competencias del perfil de egreso, tal como se observa en la tabla siguiente:

TABLA 1. Competencias específicas y sub-competencias del área de intervención en contexto de renovación curricular

\begin{tabular}{|l|l|}
\hline CE3 & $\begin{array}{l}\text { Intervenir en situaciones sociales de distintos niveles de complejidad, } \\
\text { promoviendo el desarrollo humano integral, valorando al ser humano en su } \\
\text { individualidad y en su carácter de sujeto colectivo }\end{array}$ \\
\hline SC3.1 & $\begin{array}{l}\text { Formular propuestas de intervención situadas y fundamentadas teórica, técnica } \\
\text { y políticamente. }\end{array}$ \\
\hline SC3.2 & $\begin{array}{l}\text { Aplicar los procedimientos metodológicos del Trabajo Social desde una } \\
\text { perspectiva ética de responsabilidad, compromiso y respeto a la diversidad } \\
\text { sociocultural. }\end{array}$ \\
\hline SC3.3 & $\begin{array}{l}\text { Evaluar las intervenciones sociales realizadas para mejorar los procesos y } \\
\text { resultados derivados de la práctica. }\end{array}$ \\
\hline SC3.4 & $\begin{array}{l}\text { Actuar profesionalmente de acuerdo a los principios y valores de los derechos } \\
\text { humanos. }\end{array}$ \\
\hline SC3.5 & $\begin{array}{l}\text { Desarrollar el trabajo interdisciplinario como parte constitutiva de la } \\
\text { intervención social. }\end{array}$ \\
\hline
\end{tabular}

Fuente: Proyecto Innovación Curricular Escuela de Trabajo Social, 2012. 
De esta manera y producto de las modificaciones que se implementan en la carrera a partir de la innovación curricular, se hace necesario hacer cambios en la programación y contenidos de las asignaturas de práctica, así como también en los instrumentos y didáctica que se utiliza en el trabajo directo con los estudiantes. Un proceso de transformación que no ha estado exento de dificultades, especialmente por el hecho de que la mayoría de las instituciones que acogen a nuestros estudiantes provienen de formaciones que responden a la tradición tecnológica para abordar la intervención. Es así que una de las críticas más potentes al concepto de tecnología es la que sostiene que este enfoque prescinde o ignora la reflexión en torno a los marcos normativos e ideológicos en que se mueve el conocimiento y la intervención social (Rivas, 2010). Por lo mismo, el trabajo con los estudiantes en las tutorías facilita la comprensión e interpretación de los contextos y el desarrollo de una perspectiva que implica considerar nuevos elementos en la configuración y análisis de la realidad.

Cabe mencionar que, el concepto de tecnología parte desde dos tipos de acciones: hacer y conocer. En el acto conocer existiría una modificación constante del sujeto conocedor y de sus herramientas (metodología). En el acto hacer es el objeto el que debe adecuarse. El hecho de concebir al Trabajo Social como una tecnología social significó tener una producción intelectual al servicio de la práctica, donde el concepto de 'modelo' inundó la formación y la práctica profesional. Por tanto, la noción más tradicional de la intervención social está referida a un tipo de quehacer, expresada en forma de praxis frente a las demandas de atención social especializada. Para Ander-Egg (1995), la intervención social designa un conjunto de actividades, realizadas de manera más o menos sistemática, para actuar sobre la resolución de problemas en las relaciones humanas y el fortalecimiento y la liberación del pueblo para incrementar el bienestar (IASSW, 2017). De acuerdo con Saavedra (2010), persiste en esta noción, la representación de la intervención como un hacer, tanto en el ejercicio profesional como en el desarrollo académico del Trabajo Social, y la intervención es entendida como sinónimo de práctica, o más bien dotada de una cierta quinésica, asentada en el quehacer profesional de un actante.

Esta visión del Trabajo Social puede ser debatida desde los aportes que hacen los estudios del discurso, y la convergencia entre las ciencias sociales y la lingüística. En este sentido, Saavedra (2010) afirma que la importancia del discurso en la comprensión de lo social es preeminente, y sus alcances no dejan indiferente al campo disciplinario del Trabajo Social. Sobre este aspecto e inicialmente se destacan los aportes de Carballeda (2007), quien reconoce en la intervención un artificio creado para controlar y manejar las consecuencias de la cuestión social.

El enfoque situacional surge entonces como una forma de interpretar y aproximarse a la realidad reconociendo las claves discursivas del problema social a partir de una situación. 
En tanto, el concepto de situación que en ciencias sociales es principalmente tributario del pensamiento de Matus (1987), puede entenderse desde dos perspectivas que no necesariamente son excluyentes; por una parte, como operacionalización espacio temporal del problema social, y por otra, como una evaluación multidimensional desde donde se agencia la intervención. Dicho concepto, responde a las circunstancias, contextos y significados en las que se manifiestan las actuaciones del Trabajo Social frente a demandas de solución de los problemas y conflictos de intereses que se evidencian en la sociedad (Saavedra, 2010). Por su parte, otro aspecto de este enfoque es la noción fundada que es desarrollada por Matus (2005), quien resignifica el Trabajo Social, situándose en "un horizonte de intervención que tenga como fundamento una rigurosa y compleja comprensión social"; la que, a su juicio, la concepción tecnológica no logra explicar. En definitiva, existen diversos autores en el campo del Trabajo Social que refieren al enfoque situacional (Cooper, 2001; Oliva y Millardi, 2011).

Para trabajar desde este enfoque en las prácticas implica establecer, de acuerdo al nivel curricular en el que se encuentre el estudiante, una serie de actividades y procedimientos que irán implementando los tutores de práctica durante el tiempo que se desarrolla el proceso - aproximadamente 30 semanas-, facilitando la experiencia y el aprendizaje del estudiante en relación al enfoque de intervención, puesto que la definición de la situación de intervención, requiere de la ubicación de los tejidos discursivos en los que diversos actores sociales validan sus expectativas y posiciones. Esto implica elaborar un ejercicio de operacionalización del problema social, orientado a delimitar el marco espacio-temporal en cual se hace posible el cambio social propuesto e intencionado (Saavedra, 2010). 
A continuación, se muestran, los instrumentos que se utilizan durante el proceso de práctica:

\section{TABLA 2. Instrumentos utilizados para el análisis situacional}

\begin{tabular}{|c|c|c|}
\hline Instrumento & Tema & Objetivo \\
\hline Práctico 1 & $\begin{array}{l}\text { Definiendo mis } \\
\text { expectativas }\end{array}$ & $\begin{array}{l}\text { Reflexionar sobre las expectativas en torno a: tutor/guía } \\
\text { y centro de práctica. }\end{array}$ \\
\hline Práctico 2 & $\begin{array}{l}\text { Lo que traigo a } \\
\text { mi práctica }\end{array}$ & $\begin{array}{l}\text { Identificar fortalezas y debilidades personales. actitudes } \\
\text { valores; capacidades y habilidades; conocimientos } \\
\text { capacidad y experiencia; motivación y deseo de } \\
\text { aprender. } \\
\text { Revisar proceso anterior en el caso que sea pertinente. }\end{array}$ \\
\hline Práctico 3 & $\begin{array}{l}\text { Identidad y ética } \\
\text { profesional. }\end{array}$ & $\begin{array}{l}\text { Implica determinar roles, responsabilidades y principios } \\
\text { éticos para el adecuado ejercicio profesional. }\end{array}$ \\
\hline Práctico 4 & $\begin{array}{l}\text { Contexto. } \\
\text { Marco político } \\
\text { e histórico de } \\
\text { la situación de } \\
\text { intervención. } \\
\text { Marco } \\
\text { institucional. }\end{array}$ & $\begin{array}{l}\text { Caracterizar los espacios profesionales en los que } \\
\text { se desarrolla la práctica. Incorporar la relación con } \\
\text { política pública/social en la que se enmarca el ejercicio } \\
\text { profesional. }\end{array}$ \\
\hline Práctico 5 & $\begin{array}{l}\text { Mapa y análisis } \\
\text { de Actores }\end{array}$ & Mapa y análisis de actores. \\
\hline Práctico 6 & Territorio & $\begin{array}{l}\text { Refiere a situar en términos territoriales la intervención. } \\
\text { Considera los aspectos espaciales, sociales, } \\
\text { demográficos, culturales e históricos. }\end{array}$ \\
\hline Práctico 7 & $\begin{array}{l}\text { Mapa } \\
\text { Conceptual }\end{array}$ & $\begin{array}{l}\text { Comprender y explicar desde los marcos teóricos y } \\
\text { conceptuales la(s) problemática que se aborda. Realizar } \\
\text { un mapa conceptual. }\end{array}$ \\
\hline
\end{tabular}

Fuente: Elaboración propia, 2019

Los prácticos se aplican específicamente durante el primer semestre y tienen como propósito fundamental, acompañar el proceso de vinculación entre el tutor y el estudiante, entre el estudiante y la institución, y las problemáticas sociales que se abordan. 


\section{TABLA 3. Instrumentos utilizados en el diseño y evaluación de la intervención}

\begin{tabular}{|c|c|c|}
\hline Tipo & Objetivo & Criterios de evaluación \\
\hline $\begin{array}{l}\text { Informe } 1 \\
\text { Análisis situacional y } \\
\text { plan de intervención }\end{array}$ & $\begin{array}{l}\text { Describir y analizar la } \\
\text { situación de intervención. } \\
\text { Diseñar un plan de } \\
\text { intervención. }\end{array}$ & $\begin{array}{l}\text { Describen y analizan situación de } \\
\text { intervención considerando las siguientes } \\
\text { categorías: actores, territorio, referentes } \\
\text { teóricos, contextos. } \\
\text { Incorporan perspectiva de género y } \\
\text { derechos humanos. } \\
\text { Proponen un plan de intervención y } \\
\text { estrategia de evaluación. }\end{array}$ \\
\hline $\begin{array}{l}\text { Informe } 2 \\
\text { Evaluación } \\
\text { participativa del plan } \\
\text { de intervención }\end{array}$ & $\begin{array}{l}\text { Evaluar el proceso } \\
\text { participativamente, su } \\
\text { funcionamiento, avance y } \\
\text { logro de los objetivos. } \\
\text { Se incorpora en informe } \\
\text { final. }\end{array}$ & $\begin{array}{l}\text { Las opciones a aplicar para llevar a } \\
\text { cabo esta evaluación intermedia es: } \\
\text { árbol de problemas; árbol de objetivos, } \\
\text { flujogramas, sociograma, matrices } \\
\text { reflexivas y delphi. }\end{array}$ \\
\hline $\begin{array}{l}\text { Informe } 3 \\
\text { Evaluación plan de } \\
\text { intervención }\end{array}$ & $\begin{array}{l}\text { Evaluar resultados de la } \\
\text { intervención de acuerdo } \\
\text { a matriz de planificación } \\
\text { y modelo/estrategia } \\
\text { propuesta. }\end{array}$ & $\begin{array}{l}\text { Describen el proceso de intervención y } \\
\text { evalúan los resultados de acuerdo a los } \\
\text { objetivos propuestos. } \\
\text { Analizan debilidades y fortalezas de } \\
\text { la metodología utilizada y reflexionan } \\
\text { entorno a los resultados. }\end{array}$ \\
\hline
\end{tabular}

Fuente: Elaboración propia, 2019

Los instrumentos de diseño y evaluación se aplican en momentos distintos del proceso (primer y segundo semestre), y responden a objetivos de aprendizaje de las asignaturas de práctica en sus distintos niveles. Si bien, los prácticos en tutoría facilitan el trabajo de identificación y desarrollo del análisis por parte del tutor, el informe resulta del trabajo de cada estudiante, el que también es acompañado por los/as profesionales que ejercen la labor de guía institucional. 
TABLA 4. Instrumentos de registro para propiciar la reflexión

\begin{tabular}{|l|l|l|}
\hline \multicolumn{1}{|c|}{ Tipo } & \multicolumn{1}{|c|}{ Objetivo } & \multicolumn{1}{c|}{ Descripción } \\
\hline Registros de campo & $\begin{array}{l}\text { Identificar y describir hitos del } \\
\text { proceso en la implementación } \\
\text { del plan de intervención. } \\
\text { Identificar facilitadores } \\
\text { y obstaculizadores de la } \\
\text { intervención }\end{array}$ & $\begin{array}{l}\text { Los registros de campo } \\
\text { corresponden al proceso de } \\
\text { intervención del estudiante. }\end{array}$ \\
\hline Bitácora de aprendizaje & $\begin{array}{l}\text { Reflexionar sobre el quehacer } \\
\text { profesional situado y } \\
\text { fundado. }\end{array}$ & $\begin{array}{l}\text { Se trata de un espacio en el que el } \\
\text { estudiante selecciona actividades } \\
\text { relacionadas con la intervención y } \\
\text { reflexiona en términos personales, } \\
\text { avanzando en la identificación de } \\
\text { habilidades y desafíos. }\end{array}$ \\
\hline Reflexión metacognitiva & $\begin{array}{l}\text { Reflexionar integralmente } \\
\text { respecto del proceso de } \\
\text { práctica. }\end{array}$ & $\begin{array}{l}\text { Es un momento en el que el } \\
\text { estudiante recupera aspectos } \\
\text { positivos y negativos del proceso; } \\
\text { toma conciencia respecto de sus } \\
\text { fortalezas y debilidades en torno a } \\
\text { aspectos actitudinales, cognitivos } \\
\text { y procedimentales. }\end{array}$ \\
\hline
\end{tabular}

Fuente: Elaboración propia, 2019

Los instrumentos de registro y reflexión se aplican durante todo el proceso de tutoría. Se comparten en los espacios grupales e individuales, y también se evalúan. Como tales, cumplen la función de acompañar las actividades en las prácticas, buscando instalar espacios de recuperación, reflexión y aprendizaje en los estudiantes.

\section{II.1 Niveles y momentos del proceso en la práctica profesional}

En el plan de estudio con renovación curricular, la carrera ha definido las prácticas profesionales como un "proceso gradual de formación profesional, experimentado por los estudiantes, a partir del cual se produce una apropiación progresiva de las competencias conceptuales, procedimentales y actitudinales que otorgan significado, identidad y especificidad al Trabajo Social. Este proceso se realiza en instituciones de carácter público o privado, bajo la supervisión de la Universidad y dicha Institución" (Universidad del Bío-Bío, 2015).

Los niveles de práctica existentes en la malla curricular de la carrera son: práctica profesional I, que se cursa en tercer año; práctica profesional II, en cuarto 
año y práctica profesional de quinto año, en el marco de la actividad de titulación, línea curricular, adscrita al área de intervención social, cuyo carácter es anual, tendiente a que los estudiantes comiencen el tránsito desde la formación inicial al ejercicio profesional, a través del acompañamiento tutorial.

El proceso de práctica en la carrera de Trabajo Social de la Universidad del Bío-Bío se puede configurar en al menos cuatro momentos:

\section{Presentación e inserción de los estudiantes en la institución}

Actividad que se realiza por parte del tutor de práctica asignado. En la institución se encuentra un guía, que corresponde al profesional que se hace cargo del estudiante durante el proceso al interior de la institución. Durante este periodo se realiza el encuadre de la práctica y establecen los compromisos iniciales para el desarrollo de la práctica del estudiante.

En las tutorías se conforman los grupos y establecen los tiempos para trabajar en aula. Se organizan las tareas y se presentan los instrumentos. En este caso, se entrega a cada estudiante un dossier de práctica en el cual se encuentran tanto el programa de la asignatura, como reglamentos de funcionamiento, guías de trabajo y rúbricas.

Los principales objetivos de esta etapa son:

- Identificar fortalezas y debilidades personales; actitudes valores; capacidades y habilidades; conocimientos y experiencia; motivación y deseo de aprender. Revisar proceso anterior en caso que sea pertinente.

- Revisar expectativas en torno a: tutor/guía y centro de práctica.

- Reflexionar sobre los principios éticos para el adecuado ejercicio profesional.

En este momento, los instrumentos que se aplican durante la tutoría corresponden a los primeros tres prácticos mencionados en la tabla №2.

\section{Formulación del análisis y planificación de la intervención}

Es el periodo que se sitúa entre la 3ra y 14ava semana de práctica. Este proceso se desarrolla con el apoyo de los/as tutores/as de práctica, los/as que además utilizan un set de actividades. Aquí se contempla desde el tercer práctico en adelante (ver tabla $N^{\circ} 2$ ) los que se irán desarrollando semana a semana en la tutoría. 
Durante este periodo la tutoría se centra tanto en las funciones de apoyo como educativa, iniciando el proceso del Análisis Situacional, que implica identificar preliminarmente el/los problemas sociales que se abordan desde la institución, así como las causas y soluciones que ya se están implementando. Esto significa para el estudiante iniciar la aproximación planteando interrogantes sobre el por qué y sobre qué se actúa- relevando- así mismo, el componente histórico, ético y valórico de la intervención, tomando en consideración los discursos institucionales, ya que para intervenir fundadamente lo primero es adentrarnos en las formas de nombrar y de interpretar la realidad. Lo anterior implica avanzar hacia la elaboración de instrumentos para el levantamiento de información que les permita a los estudiantes describir y analizar la situación de intervención desde la perspectiva de los actores, el territorio y los contextos, articulandose con marcos teóricos y conceptuales que expliquen la situación concluyendo en una síntesis, reflexión y juicio profesional, que busca una integración lógica de los componentes e integra las necesidades de los involucrados -dando cuenta de los problemas que serían susceptibles de intervenir- a fin de avanzar en la construcción de una propuesta de intervención situacional en la que se formulan las posibles hipótesis y estrategias para la solución. Para llegar hasta este punto el trabajo del/la tutor/a se centra en intencionar el desarrollo de actividades que se aplican durante los primeros meses y que finalizan con una síntesis sobre la situación de intervención a partir de todos los elementos e información que el estudiante levantó durante el proceso, y que además le significó involucrarse tanto con los equipos profesionales como con el material documental relacionado con el quehacer institucional.

\section{FIGURA 1. Matriz de análisis situacional}

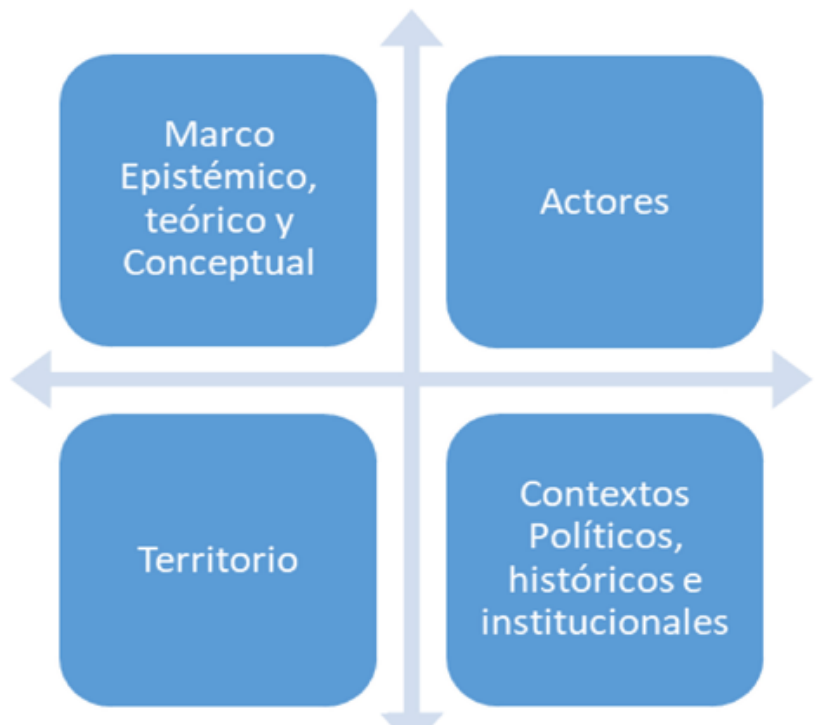

Fuente : Elaboración propia, 2019 
En esta etapa, la tutoría cobra especial relevancia ya que los estudiantes requieren procesar y reflexionar en conjunto con el tutor, quien guía el proceso que finaliza con la formulación de un juicio profesional, que se expone y discute tanto con el tutor, como con compañeros y profesionales de la institución. A partir de este momento, deviene un proceso de planificación que significa para los estudiantes, avanzar en la formulación de una matriz de planificación que pueda dar cuenta de objetivos, metodología y recursos para implementar en la siguiente etapa. Esta planificación si bien es una propuesta que surge de parte del estudiante, responde a la realidad institucional y las problemáticas/necesidades que el estudiante identifica. En consecuencia, cuenta tanto con el respaldo de su Guía de Práctica como de los equipos profesionales.

\section{Implementación y evaluación de la práctica}

Actividad que se sitúa aproximadamente entre la 15ava y 25ava semana de práctica. Etapa durante la cual la planificación propuesta se implementa por parte del estudiante y se evalúa. El trabajo de los tutores de práctica en esta etapa, básicamente es acompañar al estudiante en la resolución de obstáculos y dificultades que se van presentando, así como también encauzar el manejo técnico e instrumental que se requiere. En este momento, el tutor aplica los instrumentos de registro y reflexión, bitácora de aprendizaje e instrumentos de evaluación. Paralelamente, se llevan a cabo visitas a terreno a fin de observar actividades en las que el estudiante participa e intenciona como parte de su plan de trabajo en su centro de práctica..

\section{Cierre y despedida de la práctica}

Periodo durante el cual el estudiante, tutor y guía profesional, inician los procesos de cierre y despedida del centro.

Durante el periodo que dura el proceso de práctica, no solo se establece un vínculo entre el tutor y el estudiante, sino que también se establecen relaciones con las instituciones de acogida, y sus proyectos, y/o programas. En este caso, los guías de práctica son actores que también aportan con su experiencia y conocimiento experto a la formación del estudiante. Sin embargo, para los profesionales de la institución el enfoque situacional de la intervención les parece ajeno a las lógicas que habitualmente se desarrollan en los programas públicos, como modelo que permite comprender y explicar la realidad en el marco de la intervención profesional. En este escenario, el tutor de práctica desempeña el rol de mediador, facilitando el tránsito de los estudiantes, haciendo las veces de puente entre la concepción tradicional de la intervención -ciclo tecnológico- y el enfoque situacional. Esto genera un desafío constante frente a los pares y profesionales 
que son parte de la red de instituciones con las que se establecen los convenios de práctica.

\section{II.2 Rol y fases de la tutoría académica en las prácticas profesionales}

Tirado, Miranda y Sánchez (2007) establecen que la calidad educativa y su promoción en los contextos universitarios depende de cómo ocurre la acción del ejercicio docente en el aula porque deriva de cómo se ejerce la enseñanza; por ende Guzmán (2011), afirma que la enseñanza educativa es un acto complejo y multidimensional, por ser una tarea en la que de manera propositiva y deliberada se pretende que alguien aprenda de otro.

Desde estos antecedentes, Anabalón, Concha y Mora (2018), aluden a que la tutoría académica actúa como el soporte elemental de la calidad educativa bajo un asesoramiento metodológico permanente que realizan los tutores académicos con los estudiantes, contribuyendo al desarrollo integral de éstos en la praxis profesional; potenciando y reforzando de manera positiva las fortalezas y, al mismo tiempo, visibilizando y superando las debilidades. A partir de ello, el docente-tutor debe actuar como facilitador del proceso de aprendizaje en el desarrollo de las tutorías académicas, entendiendo éstas como "una actividad de carácter formativo que incide en el desarrollo integral de los estudiantes universitarios en su dimensión intelectual, académica, profesional y personal" (Ferrer, 2003, p.67).

La tutoría académica es fundamental en las asignaturas de práctica profesional porque los estudiantes establecen un vínculo con el docente-tutor académico, a través de quien se canaliza esta acción educativa personalizada de acompañamiento, asesoramiento y apoyo en la adquisición y maduración de las competencias y en la configuración del proyecto personal y profesional del estudiante (Lobato y Guerra, 2014; Rodríguez-Hoyos, Calvo y Haya, 2015; AmorAlmedina, 2016; Harvey, Ambler y Cahir, 2016).

La formación inicial de los estudiantes de la Escuela de Trabajo Social está caracterizada por el desarrollo de habilidades duras y blandas en permanente reflexión crítica, fundada y reflexiva. Igualmente, el autoaprendizaje cobra relevancia en la capacitación del individuo para la vida profesional, a partir de los ámbitos constitutivos del ser humano. De acuerdo con lo anterior, es que se pone de manifiesto la importancia de desarrollar tutorías académicas en educación superior, en la que se deje de lado la concepción tradicional de la tutoría, centrada solo en resolver dudas de una asignatura específica, a fin de dar cabida a una tutoría más amplia, de carácter orientador, que incluya la vida académica de los estudiantes de la Escuela de Trabajo Social. 
En cuanto a las fases de la tutoría, éstas surgen a partir de diversas jornadas de reflexión, sostenidas por el equipo de docentes-tutores de práctica de la Escuela de Trabajo Social y su descripción se realiza desde un enfoque centrado tanto en el aprendizaje del estudiante como en la enseñanza, relevando el rol del docente-tutor.

\section{Fase 1. Centrada en el aprendizaje: reflexión, integración y resignificación de la praxis}

\section{a) Aproximación al proceso de intervención/actuación profesional}

La actividad reflexiva se constituye en una estrategia de aprendizaje, monitoreo y seguimiento cíclico del proceso de práctica. Significa reflexionar permanentemente en torno a la integración teórico-práctica y al proceso metodológico del Trabajo Social, para identificar, sistematizar, deconstruir y evaluar los aprendizajes logrados.

Los estudiantes en esta fase, se sitúan en contexto y área de desempeño profesional; reconocen marcos conceptuales, epistemológicos y/o teóricos que están presentes en la intervención/actuación profesional, así como los recursos institucionales existentes y a los que se podría acceder; identifican y problematizan las necesidades de los sujetos; reflexionan en torno a su rol como estudiantes en el espacio profesional; reconocen los recursos personales, necesidades y expectativas de aprendizaje en torno al proceso de práctica; y aplican técnicas y metodologías para aproximarse al contexto institucional o territorial.

\section{b) Construcción propuesta de intervención/actuación profesional}

En esta fase el estudiante reflexiona permanentemente en relación al contexto institucional, al área de desempeño profesional, a los sujetos de atención, a la propuesta de intervención/actuación y a su rol como agente de intervención. Las principales acciones son: identificación y evaluación de los aprendizajes relevantes en torno a lo anterior (metacognición = qué he aprendido). Se espera que el estudiante genere una propuesta creativa que responda a las necesidades identificadas a partir del conocimiento institucional o del espacio territorial, y que problematice las necesidades reconocidas en su aproximación al ámbito de intervención/actuación profesional.

Se identifican y reflexiona sobre las posibles propuestas de intervención/ actuación en torno a las necesidades detectadas (el qué); se define, describe y 
fundamenta epistemológica-teórica, metodológica y prácticamente la propuesta elegida (por qué); se identifican los niveles de intervención que se abordarán en la propuesta (individuos, familias, grupos, comunidades y/o territorios); se definen los objetivos, metas, actividades, metodologías, técnicas, recursos, plazos, tipos y modelos evaluación asociados a la propuesta y a otras intervenciones/actuaciones profesionales (para qué, cómo, con qué); se caracteriza a los sujetos de intervención/actuación de la propuesta (para quién y con quién); y se fundamenta la implementación de la propuesta.

\section{Fase 2. Centrada en la enseñanza: reflexión, integración y resignificación de la praxis}

\section{a) Aproximación al proceso de intervención/actuación profesional}

En esta fase se produce un primer acercamiento con los estudiantes en tutorías de práctica; se realiza un encuadre respecto a lo que significa insertarse en un espacio institucional o territorial; se rescata, reformula y/o refuerzan sus ideas previas y expectativas respecto a las prácticas; se reflexiona en torno al concepto de intervención/actuación profesional; se revisan los marcos conceptuales, epistemológicos y/o teóricos que subyacen a la intervención/actuación profesional; se activan conocimientos previos; se contextualizan los conocimientos en la práctica y se ejercitan las técnicas de actuación profesional. Asimismo, se facilitan en las tutorías de práctica instancias de reflexión en torno a la integración teórico-práctica y al proceso metodológico del Trabajo Social, para identificar, sistematizar, deconstruir, evaluar los aprendizajes logrados y desarrollar acciones de mejora pertinentes.

\section{b) Construcción propuesta de intervención/actuación profesional}

Esta fase permite revisar elementos técnicos para la construcción de la propuesta; facilitar instancias de reflexión; retroalimentar; activar conocimientos previos de tipo metodológicos y teóricos para su construcción; demostrar la relevancia de la conexión entre la propuesta de intervención y los marcos conceptuales, epistemológicos y/o teóricos que están a la base; revisar y retroalimentar los análisis que realiza el/la estudiante sobre su vinculación con los marcos conceptuales, epistemológicos y/o teóricos. 


\section{Conclusiones}

Considerando el objetivo de este trabajo, se concluye que a partir de la innovación curricular, se hace necesario realizar cambios en los programas y contenidos de las asignaturas de prácticas, instrumentos y didáctica que se utiliza en el trabajo directo con los estudiantes, al cual se ha denominado como tutoría académica, a través de la cual se acompaña continuamente el proceso formativo y se facilita la comprensión e interpretación de los contextos, así como el desarrollo de una perspectiva con elementos idóneos para la configuración y análisis de la realidad. Es así, que la tutoría académica, como estrategia formativa, permite entender el enfoque situacional como una perspectiva que proporciona una interpretación y aproximación a la realidad, reconociendo las claves discursivas del problema social a partir de una situación en particular.

Las asignaturas de práctica, de acuerdo al nivel curricular en el que se encuentre el estudiante, implican una serie de actividades y procedimientos que van implementando los docentes-tutores de práctica durante el tiempo que se desarrolla el proceso (entre 28 y 30 semanas), facilitando la experiencia y el aprendizaje del estudiante en relación al enfoque de intervención.

Los instrumentos iniciales que se aplican en el proceso tutorial tienen como propósito fundamental, acompañar la vinculación entre el docente-tutor y el estudiante, entre el estudiante y la institución, y el acercamiento hacia las problemáticas sociales que se abordan. Posteriormente los instrumentos de diseño y evaluación que se llevan a cabo en distintos momentos del proceso, responden a los objetivos de aprendizaje de las asignaturas de práctica en sus distintos niveles. En este contexto, los prácticos desarrollados facilitan el trabajo de identificación y desarrollo del análisis por parte del docente-tutor, siendo el informe de práctica resultado del trabajo de cada estudiante, el que también es acompañado por los/as profesionales que ejercen la labor de guía institucional en los respectivos centros o instituciones. Por último, los instrumentos de registro y reflexión se comparten en las instancias grupales e individuales, cumpliendo la función de acompañar las actividades en las prácticas e instalar espacios de retroalimentación, metacognición y aprendizaje en los estudiantes.

Se destaca que la formación académica y profesional de los estudiantes se caracteriza por una formación en competencias genéricas y específicas para el ejercicio del trabajo social de acuerdo al perfil de egreso que busca instalar capacidades en los estudiantes que se orientan hacia la reflexión crítica, fundada y reflexiva; como asimismo en el autoaprendizaje, poniendo de manifiesto la importancia de desarrollar tutorías que sean parte de la actividad académica de calidad en la educación superior.

Es así que Cano (2009) menciona que la tutoría debiera ser el elemento básico de calidad educativa, expresada en sus diversas formas, sea ésta, académica, 
personal y/o profesional; como asimismo en sus diferentes manifestaciones individual, grupal y entre pares; todas ellas complementarias entre sí. Esta perspectiva deja de lado la concepción tradicional de la tutoría, centrada sólo en resolver dudas de una asignatura específica, para pasar a una tutoría más amplia, de carácter orientador, que incluye la vida académica de los estudiantes de la Escuela de Trabajo Social, y necesaria para avanzar en los cambios y transformación que desde el año 2013 se instala en la carrera como marco de la innovación curricular.

Los cambios curriculares e incorporación de nuevos enfoques en la formación para la intervención profesional han implicado asimismo hacer un cambio en la forma de pensar e interpretar la realidad, reconociendo en los discursos las claves para aproximarnos a las problemáticas sociales en tanto pluralidad, alteridad y territorialidad.

Es en este contexto que, las tutorías académicas se han constituido en una instancia académica de alta exigencia y nivel formativo en el ámbito de las prácticas profesionales de los estudiantes de la Carrera, implicando un proceso permanente de acompañamiento y asesoramiento metodológico, siendo un soporte elemental de la calidad educativa que contribuye a una adecuada inserción laboral y al desarrollo integral de los futuros Trabajadores y Trabajadoras Sociales en las diversas áreas del desempeño profesional, pues tal como mencionan Martínez, Pérez y Martínez (2018, p.637) "la tutoría se convierte en un elemento clave de calidad en cuanto crea valor añadido para los estudiantes, como uno de los principales clientes de la universidad, atendiendo a sus necesidades o carencias de un modo globalizado".

Cabe destacar que en las tutorías académicas se potencian y refuerzan de manera positiva las fortalezas del estudiantado y, al mismo tiempo, visibilizan y superan las debilidades observadas en la formación profesional; facilitando la transmisión cultural y contribución al desarrollo de la investigación y la intervención profesional, formando profesionales capaces de desenvolverse en un escenario, cambiante y complejo, que exige el desempeño de múltiples roles, el compromiso constante y la responsabilidad ética en el ejercicio profesional.

De acuerdo a lo anterior, surge como desafío poder complementar este proceso con investigaciones que den cuenta de la percepción de los estudiantes, docentes-tutores, guías institucionales y otros actores involucrados, a fin de evaluar y perfeccionar la tutoría académica en función de los posibles nudos críticos o debilidades que surjan y de esta forma avanzar hacia el mejoramiento continuo, especialmente en la articulación teórico-práctica, las estrategias pedagógicas y didácticas, para apoyar de manera sistemática y pertinente a los estudiantes en su tránsito por la formación profesional e identidad profesional. 


\section{Referencias bibliográficas}

Amor-Almedina, M. (2016). Evaluación de la Orientación y la Tutoría en la Facultad de Ciencias de la Educación de la Universidad de Córdoba. Educatio Siglo XXI, 34, 93-112. doi: http://dx.doi.org/10.6018/j/253231

Anabalón, Y., Concha, M. y Mora, M. (2018). Tutorías académicas y Prácticas Profesionales: invitación al desempeño profesional inicial en la formación trabajadores sociales. UCMaule Revista académica, 54(1), 51-64. doi: 10.29035/ucmaule.54.51

Ander-Egg, E. (1995). Diccionario de Trabajo Social. Buenos Aires: Lumen.

Bernasconi, A. y Rojas, F. (2004). Informe sobre la educación superior en Chile: 1980- 2003. Santiago, Chile: Editorial Universitaria.

Cano, R. (2009). Tutoría universitaria y aprendizaje por competencias. ¿Cómo lograrlo? Revista Electrónica Interuniversitaria de Formación del Profesorado, 12(1), 181-204.

Carballeda, A. (2007). La intervención en lo social. Exclusión e integración en los nuevos escenarios sociales. Buenos Aires: Paidós.

Consejo de Rectores de las Universidades Chilenas [CRUCH]. (2012). Innovación Curricular en las Universidades del Consejo de Rectores. Reflexiones y procesos en las Universidades del Consejo de Rectores. Prácticas Internacionales. Editorial Universidad de Valparaíso.

Consejo de Rectores de las Universidades Chilenas [CRUCH]. (2013). Manual para la implementación de Sistema de Créditos Académicos Transferibles. SCTChile. Santiago, Chile: Consejo de Rectores de las Universidades Chilenas.

Cooper, B. (2001). Constructivism in Social Work. British Journal of social work, 31, $721-738$.

Delors, J. (1996). La educación encierra un tesoro. Informe a la UNESCO de la Comisión internacional sobre la educación para el siglo XXI. Madrid: Santillana/UNESCO.

Ferrer, V. (2003). La acción tutorial en la Universidad. En Michavila, F. y García, J. (coord.). La Tutoría y los nuevos modelos de aprendizaje en la Universidad. Madrid: Consejería de Educación de la Comunidad Autónoma de Madrid/ Cátedra UNESCO de Gestión Política Universitaria de la Universidad Politécnica de Madrid, (pp. 67-84). 
Guzmán, J. C. (2011). La calidad de la enseñanza en educación superior ¿Qué es una buena enseñanza en este nivel educativo? Perfiles educativos, 33, 129141.

Harvey, M., Ambler, T., y Cahir, J. (2016). Spectrum approach to mentoring: An evidenced based approach to mentoring for academics working in higher education. Teacher Development, 21(1), 160-174. doi: 10.1080/13664530.2016.1210537

Inostroza, R., Zúñiga, R. y Ayala, M. (2013). La formación por competencias y los estudiantes: confluencias y divergencias en la construcción del docente ideal. Calidad en la educación, (38), 277-304. doi: 10.4067/S07184565201300010000

International Association of Schools of Social Work (IASSW). (2017). La Asociación Internacional de Escuelas de Trabajo Social: Una Breve Historia de su Fundación y sus Primeros Años. Recuperado de https://www.iassw-aiets. org/la-asociacion-internacional-de-escuelas-de-trabajo-social-una-brevehistoria-de-su-fundacion-y-sus-primeros-anos/

Lobato, C. y Guerra, N. (2014). Las tutorías universitarias en el contexto europeo. Orientación y sociedad, Revista internacional e interdisciplinaria de orientación vocacional ocupacional, 14, 1-23.

Martínez, P., Pérez, F.J., y Martínez, M. (2018). Aplicación de los Modelos de Gestión de Calidad a la Tutoría Universitaria. Revista Complutense de Educación, 29(3), 33-50.

Matus, C. (1987). Política, Planificación y Gobierno. Caracas: Fundación ALTADIR. Instituto Latinoamericano y del Caribe de Planificación Económica y Social.

Matus, T. (2005). Propuestas contemporáneas en trabajo social: hacia una intervención polifónica. Buenos Aires: Espacio.

Organización de Cooperación y Desarrollo Económicos [OCDE]. (1998). La educación en una mirada: indicadores OCDE 1998. Comisión económica para América Latina y El Caribe. Recuperado de https://www.cepal.org/prensa/ noticias/comunicados/5/275/expobajraj.htm

Oliva, A. y Mallardi, M. (Coord.). (2011). Aportes táctico operativos a los procesos de intervención del Trabajo Social. Tandil: UNICEN.

Pey, R., Chauriye, S., y Chiuminatto, P. (2018). Formación general en la Universidad de Chile. Calidad en la Educación, (17), pp. 39-49. doi: 10.31619/caledu. n17.405 
Universidad del Bío-Bío. (2012). Proyecto Innovación Curricular Escuela de Trabajo Social. Concepción: Universidad del Bío-Bío.

Universidad del Bío-Bío. (2015). Reglamento de Prácticas Escuela de Trabajo Social. Concepción: Universidad del Bío-Bío.

Rollin, K. (1997). Los temas críticos de la Educación Superior en América Latina en los años 90. Volumen 2. México: ANUIES.

Rivas, R. (2010). El Trabajo Social como Tecnología Social y Disciplina. Revista Margen, (57), 1-10.

Rodríguez-Hoyos, C., Calvo, A. y Haya, I. (2015). La tutoría académica en la educación superior. Una investigación a partir de entrevistas y grupos de discusión en la Universidad de Cantabria (España). Revista Complutense de Educación, 26(2), 467-481. doi: 10.5209/rev_RCED.2015.v26.n2.43745

Saavedra, J. (2010). Configuración discursiva en intervención social fundada, situada y reflexiva. TS Cuadernos de Trabajo Social, (6), 57-70.

Tardif, J. (2003). Développer un programme par compétences: de l'intention à la mise en oeuvre. Pedagogie Collegiale, 16(3), 36-44.

Tejada, J. (2002). El docente universitario ante los nuevos escenarios: implicaciones para la innovación docente. Acción Pedagógica, 11(2), 30-42.

Tirado, F., Miranda, A. y Sánchez, A. (2007). La evaluación como proceso de legitimidad: la opinión de los alumnos. Reporte de una experiencia. Perfiles educativos, 29(118), 7-24.

Dirección de correspondencia:

Yasna Anabalón

Trabajadora Social, Magister en familia con mención en mediación familiar y Candidata a Doctora en educación en Consorcio, perteneciente de la Universidad del Bío-Bío. Becaria CONICYT/ Doctorado Nacional/ 2017/ Folio 21170133. Tutora de práctica de la Escuela de Trabajo Social, Universidad del Bío-Bío, Chillán, Chile.

Contacto:

yanabalon@ubiobio.cl 